\section{JTI}

JOURNAL OF

TRAUMA AND INJURY

\title{
Transosseous Tie Fixation Using Krackow Sutures and Bone Tunnels in a Comminuted Fracture of the Upper Pole of the Patella: A Case Report
}

\author{
Jong Seok Baik, M.D.
}

Department of Orthopedic Surgery, Wonkwang University Hospital, Wonkwang University School of Medicine, Iksan, Korea

Received: December 21, 2020

Revised: February 17, 2021

Accepted: March 11, 2021

\section{Correspondence to}

Jong Seok Baik, M.D.

Department of Orthopedic Surgery, Wonkwang University Hospital,

Wonkwang University School of Medicine, 895 Muwang-ro, Iksan 54538, Korea

Tel: $+82-63-859-1360$

Fax: $+82-63-852-9329$

E-mail: pis0408@naver.com

ORCID: https://orcid.org/0000-0002-

1877-1837
Comminuted fractures of the patella mostly occur at the inferior pole and require appropriate reduction and fixation to restore the extensor mechanism. Conventional methods such as tension-band wiring are not enough to gain proper fixation strength. Numerous methods have been reported, including circumferential cerclage wiring, osteosynthesis, and suture anchors depending on the fracture pattern. Herein, the author reports a relatively rare case of a comminuted fracture of the upper pole of the patella, for which we used augmented Krackow sutures in the quadriceps and fixation with tying of the suture limbs through patellar bone tunnels. Satisfactory results were obtained in terms of reduction and extensor mechanism recovery.

Keywords: Patella; Fractures, comminuted; Transosseous fixation

\section{INTRODUCTION}

Patella fractures account for $0.5-1.5 \%$ of all skeletal fractures. Indications for operative treatment include displacement of more than $3 \mathrm{~mm}$, articular displacement of more than $2 \mathrm{~mm}$, and extensor mechanism failure [1]. Anatomical reduction and rigid fixation are important due to the functional importance of the patella. Conventional tension band wiring is insufficient for appropriate reduction and stability for comminuted fractures [2]. Therefore, various methods including circumferential cerclage wiring, osteosynthesis using plates, fixation using suture anchors, and transosseous fixation have been proposed $[3,4]$. Most reports, however, dealt with sleeve avulsion or comminuted fractures of the inferior pole. We hereby report an uncommon case (http://creativecommons.org/licenses/by-nc/4.0/) which permits unrestricted noncommercial use, distribution, and reproduction in any medium, provided the original work is properly cited. 


\section{JTI}

of a comminuted fracture of the upper pole of the patella, in which bone union and recovery of the extensor mechanism were achieved without any complications through Krackow sutures and transosseous bone tunnels.

This study was approved by Institutional Review Board of Wonkwang University Hospital (IRB No. 2020-12-025).

\section{CASE REPORT}

A 43-year-old male patient visited Wonkwang University Hospital emergency room due to pain in the right knee caused by a motorcycle accident. A physical examina- tion showed swelling, limitation of extension of the right knee, and tenderness of the anterior knee. There were no neurological abnormalities or open wounds. An X-ray showed fracture and displacement at the upper pole of the patella. On 3-dimensional computed tomography and magnetic resonance imaging (Ingenia 3.0T, Philips, Eindhoven, Netherlands), a comminuted fracture of the upper pole of the patella and displacement more than $10 \mathrm{~mm}$ due to traction by the quadriceps were found. There were no ligamentous or tendinous injures (Fig. 1). We planned operative treatment for the fracture. Laboratory findings were normal except for a slight increase in the C-reactive protein level.
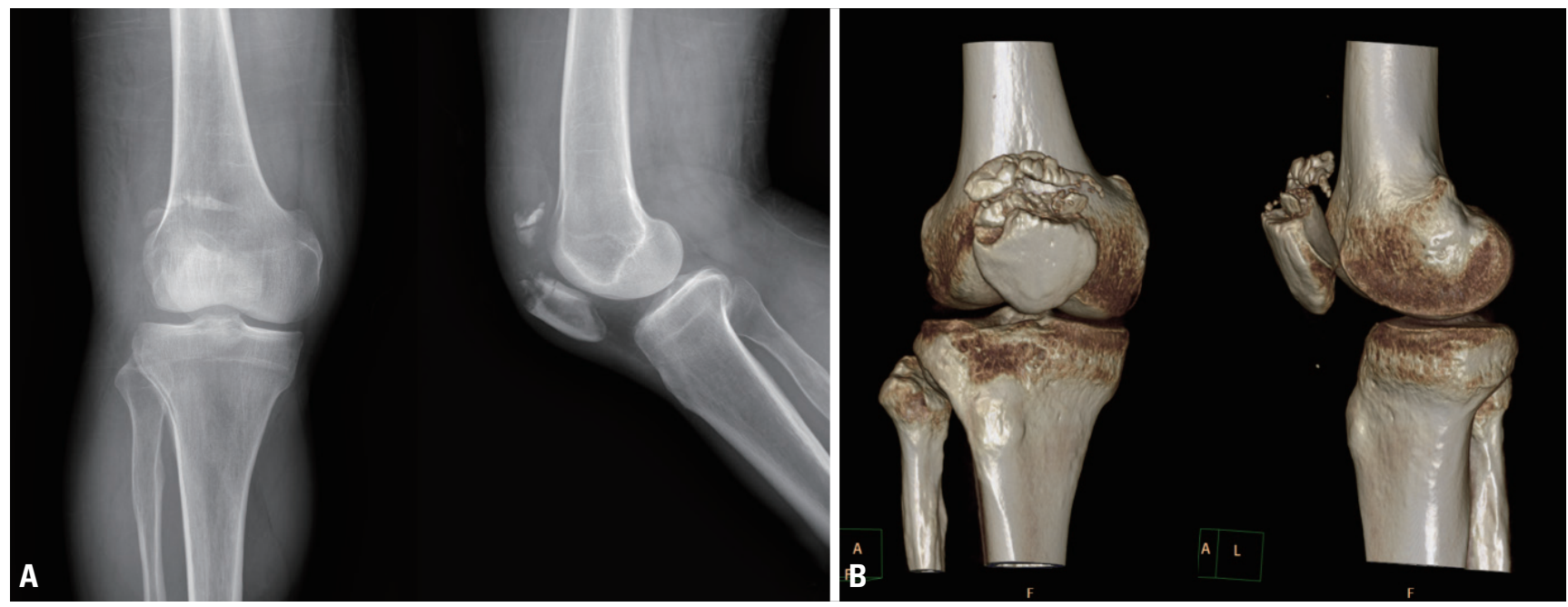

Fig. 1. (A) Preoperative X-rays of a comminuted and displaced fracture of the upper pole of the patella. (B) Preoperative 3-dimensional computed tomography finding a severely comminuted fracture.
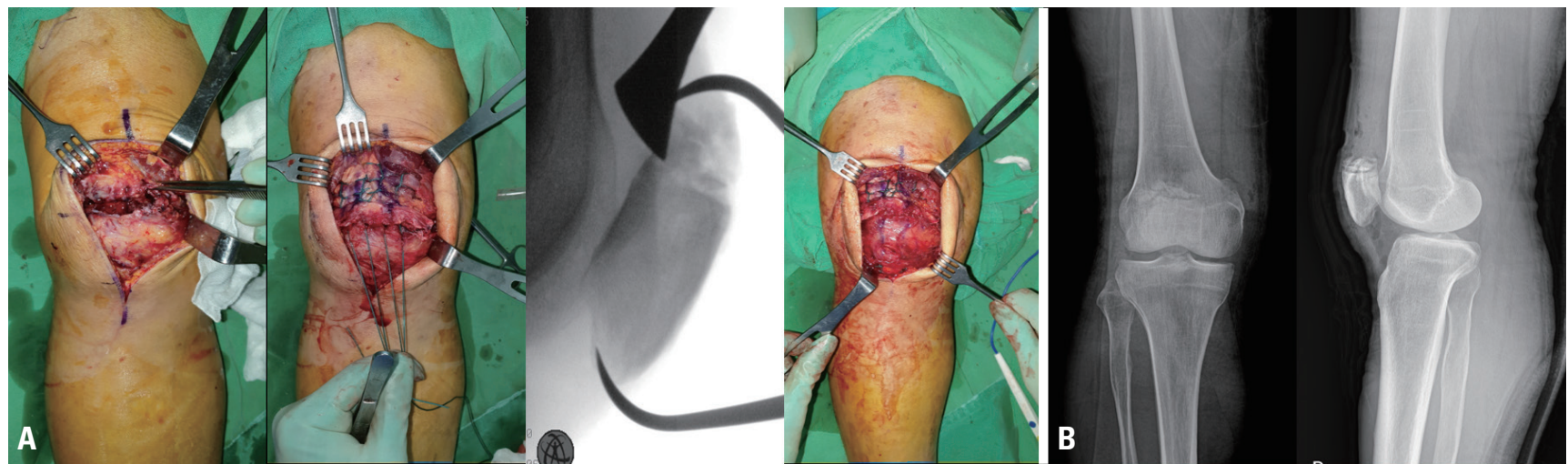

Fig. 2. (A) Intraoperative findings of comminution and displacement of the fragment. The reduction was maintained using Krackow sutures and a reduction forceps, the suture limbs were tied, and the retinaculum was repaired. (B) Postoperative X-rays showed reduction without displacement. 
Under spinal anesthesia and supine position, a tourniquet was applied at the proximal thigh. A longitudinal incision was made along the anterior midline to expose the patella and a hematoma was removed at the fracture site, with care taken to avoid soft tissue injuries. The fragments were comminuted and displaced to the proximal side, being attached to the quadriceps. We decided that conventional tension-band wiring would not be appropriate for maintaining reduction. Therefore, we planned to repair the quadriceps and to perform reduction using the patellar bone tunnel. After identifying the location of the fragment, we performed 4-strand double Krackow locking sutures with Ethibond \#5 on the quadriceps. Then, we used a $2.5-\mathrm{mm}$ drill bit to make three vertical transosseous bone tunnels in the patella. After passing two central suture limbs of the Krackow loop through the central bone tunnel, we passed the medial and lateral limbs through the medial and lateral tunnels, respectively. Then, minimizing entrapment of the soft tissue, we pulled the suture limbs under guidance of an image intensifier to reduce the fragment, maintained the anatomical reduction with reduction forceps, and made ties under the patella (Fig. 2). After the retinacular tears were repaired using \#1 Vicryl sutures, we used the image intensifier to confirm that there was no separation of the fragment

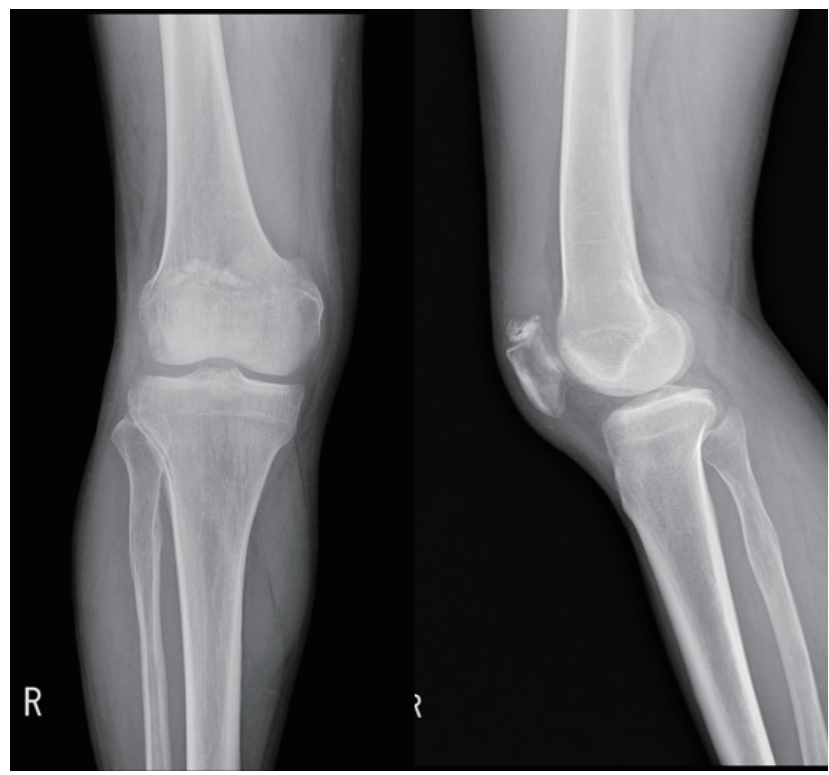

Fig. 3. At 3 months after surgery, an X-ray examination showed bone union progression without displacement of the bone fragment. during full flexion and extension of the knee to check the stability of the fixation. From the first day after surgery, the patient started quadriceps muscle strength exercises and partial weight-bearing with a crutch using a full-extension brace. The range of motion of the joint was initially $30^{\circ}$ at 2 weeks later, and then increased by $30^{\circ}$ every 2 weeks. At follow-up after 3 months after surgery, the patient showed extensor mechanism recovery and no limitation in the range of motion, which made it possible for him to perform full weight-bearing activities and return to everyday life without a brace (Fig. 3).

\section{DISCUSSION}

The goals of operative treatment of a patellar fracture are anatomical reduction of the fragment, recovery of the extensor mechanism, and stable fixation, which allows early joint movement [5]. Traditionally, the displaced fragment is reduced using tension-band wiring. In cases of comminuted fractures, this method usually cannot offer appropriate fixation strength, which has prompted advances in various methods such as cerclage wiring and plates. Furthermore, due to concerns regarding implant-related complications, ongoing research has investigated metal-free techniques. There have been some reports regarding satisfactory results with fewer complications in patients who underwent metal-free treatments than in those who received metal fixations [6,7]. Most studies have dealt with inferior comminuted fractures of the patella or fractures in skeletally immature children, whereas few have investigated the treatment of comminuted fractures of the upper pole of the patella. We contemplated the surgical method in this case of comminuted fracture where precise anatomical reduction was difficult, and there was a possibility of impairment of the extensor mechanism due to shortening of the quadriceps. We achieved a satisfactory result using transosseous fixation, which does not provoke implant-induced irritation or migration of the implant and does not require additional surgery for hardware removal. In patellar fractures, understanding the advantages and disadvantages of various surgical methods and choosing an appropriate method according to the precise pattern of the fracture based on a physical and 
radiological examination, satisfactory results with fewer complications can be achieved.

\section{CONFLICTS OF INTEREST}

No potential conflict of interest relevant to this article was reported.

\section{INFORMED CONSENT}

This type of study does not require informed consent.

\section{ACKNOWLEDGEMENTS}

This research was supported by grant from Wonkwang University, 2021.

\section{REFERENCES}

1. Galla M, Lobenhoffer P. Patella fractures. Chirurg 2005;76:98799.

2. Lee BJ, Chon J, Yoon JY, Jung D. Modified tension band wiring using FiberWire for patellar fractures. Clin Orthop Surg 2019;11:244-8.

3. Cho JW, Kim J, Cho WT, Gujjar PH, Oh CW, Oh JK. Comminuted inferior pole fracture of patella can be successfully treated with rim-plate-augmented separate vertical wiring. Arch Orthop Trauma Surg 2018;138:195-202.

4. Kim KS, Suh DW, Park SE, Ji JH, Han YH, Kim JH. Suture anchor fixation of comminuted inferior pole patella fracture-novel technique: suture bridge anchor fixation technique. Arch Orthop Trauma Surg 2020 Oct 30. doi: 10.1007/s00402-020-036715 [Epub ahead of print].

5. Melvin JS, Mehta S. Patellar fractures in adults. J Am Acad Orthop Surg 2011;19:198-207.

6. Chatakondu SC, Abhaykumar S, Elliott DS. The use of non-absorbable suture in the fixation of patellar fractures: a preliminary report. Injury 1998;29:23-7.

7. Gosal HS, Singh P, Field RE. Clinical experience of patellar fracture fixation using metal wire or non-absorbable polyester--a study of 37 cases. Injury 2001;32:129-35. 